\section{Adsorption of a Polymer to a Randomly Interacting Surface}

\section{THEO ODIJK}

Department of Polymer Technology,

Faculty of Chemical Engineering and Materials Science, Delft University of Technology, P.O. Box 5045,

2600 GA Delft, The Netherlands. Received May 25, 1989;

Revised Manuscript Received August 21, 1989

Consider a surface that is highly irregular, physically or chemically, so much so that the nonuniformity must be regarded as a stochastic variable. Such a surface would interact randomly with every link of a polymer chain. Conceivably, circumstances could be controlled so that on average this stochastic interaction is exactly zero. Nevertheless, the macromolecule would be attracted to the surface. In fact, the interaction as such is not the quantity of interest, for it has to be weighted by the probability, which is bigger for attraction than for repulsion. Hence, a long enough chain is expected to be adsorbed, at least under certain conditions. But it is unlikely to be localized within a minute region of the surface-on the order of a surface correlation length squaredbecause the chain is allowed to sample the adjoining threedimensional space. This contrasts with the behavior of an ideal polymer chain trapped in a certain kind of random medium in which it has been argued to be fully localized. $^{1,2}$

We investigate the adsorption of a polymer to a random surface by first regarding the general problem of an ideal chain perturbed by a Gaussian random field $\phi(\mathbf{r}, n)$ depending on position $\mathbf{r}$ and link $n$. The dependence on $n$ is formal since it is nonexistent for the problem at hand. The averaging is effected over $\phi$, whose stochastic properties are determined by two moments only

$$
\begin{gathered}
\langle\phi(\mathbf{r}, n)\rangle=0 \\
\left\langle\phi(\mathbf{r}, n) \phi\left(\mathbf{r}^{\prime}, n^{\prime}\right)\right\rangle=\Gamma\left(\mathbf{r}, n, \mathbf{r}^{\prime}, n^{\prime}\right)
\end{gathered}
$$

where the function $\Gamma$ will be specified below. If the external stochastic field is weak, the probability $G\left(\mathbf{r}, n, \mathbf{r}_{0}, 0\right)$ of a chain of $n$ links each of length $a$ starting at $\mathbf{r}_{0}$ and ending at $\mathbf{r}$ satisfies a diffusion equation ${ }^{7}$

$$
L G \boxminus \frac{\partial G}{\partial n}-\frac{1}{6} a^{2} \Delta G+\phi G=\delta(n) \delta\left(\mathbf{r}-\mathbf{r}_{0}\right)
$$

On averaging this expression

$$
\frac{\partial\langle G\rangle}{\partial n}-\frac{1}{6} a^{2} \Delta\langle G\rangle+\langle\phi G\rangle=\delta(n) \delta\left(\mathbf{r}-\mathbf{r}_{0}\right)
$$

we discover that the crux is to rewrite $\langle\phi G\rangle$ in terms of $\langle G\rangle$, which can be done approximately via a method from the theory of Gaussian random fields. ${ }^{3}$

First we functionally differentiate eq 3 with respect to $\phi\left(\mathbf{r}^{\prime}, n^{\prime}\right)$

$$
L\left(\frac{\delta G\left(\mathbf{r}, n, \mathbf{r}_{0}, 0\right)}{\delta \phi\left(\mathbf{r}^{\prime}, n^{\prime}\right)}\right)=-\delta\left(n-n^{\prime}\right) \delta\left(\mathbf{r}-\mathbf{r}^{\prime}\right) G\left(\mathbf{r}, n, \mathbf{r}_{0}, 0\right)
$$

Since $G$ is the Green function of the operator $L$, we have

$$
\left\langle\frac{\delta G\left(\mathbf{r}, n, \mathbf{r}_{0}, 0\right)}{\delta \phi\left(\mathbf{r}^{\prime}, n^{\prime}\right)}\right\rangle=-\left\langle G\left(\mathbf{r}, n, \mathbf{r}^{\prime}, n^{\prime}\right) G\left(\mathbf{r}^{\prime}, n^{\prime}, \mathbf{r}_{0}, 0\right)\right\rangle
$$

Next, the Furutsu-Novikov identity ${ }^{3}$ valid for Gaussian stochastic fields enables us to obtain the desired moment

$$
\begin{aligned}
& \left\langle\phi(\mathbf{r}, n) G\left(\mathbf{r}, n, \mathbf{r}_{0}, 0\right)\right\rangle= \\
& -\iint \mathrm{d} \mathbf{r}^{\prime} \mathrm{d} n^{\prime} \Gamma\left(\mathbf{r}, n, \mathbf{r}^{\prime}, n^{\prime}\right)\left\langle G\left(\mathbf{r}, n, \mathbf{r}^{\prime}, n^{\prime}\right) G\left(\mathbf{r}^{\prime}, n^{\prime}, \mathbf{r}_{0}, 0\right)\right\rangle
\end{aligned}
$$

In eqs 4 and 7 the second moment $\langle G G\rangle$ is related to $\langle G\rangle$. It is also possible to establish a hierarchy of equations connecting the second moment to moments of higher order. Here, we trivially break the hierarchy by employing an approximation of the Hartree type $\langle G G\rangle \simeq$ $\langle G\rangle\langle G\rangle$. This procedure is on a par with the classical self-consistent field theory for the excluded-volume effect ${ }^{4}$ (recall that this theory can be rewritten in terms of a complex random field).

Next, we specify the stochastic interaction between the surface and a link. The averaging over the random field $\phi$ is viewed as equivalent to an average over an ensemble of stochastically irregular surfaces. The $z$ axis in our Cartesian coordinate system is normal to the surface, which is located at $z=0$. Note that we view the surface as perfectly flat; its physical or chemical properties vary irregularly within the plane of the surface. If the range $\xi_{8}$ of the correlation of the random interaction within the plane is much shorter than the bulk correlation length $\xi_{\mathrm{b}}$ and the length scale $z_{0}$ defined below, we may write

$$
\Gamma\left(\mathbf{r}, n, \mathbf{r}^{\prime}, n^{\prime}\right)=V\left(z, z^{\prime}\right) \xi_{\mathrm{B}}{ }^{2} \delta\left(x-x^{\prime}\right) \delta\left(y-y^{\prime}\right)
$$

The dimensionless function $V\left(z, z^{\prime}\right)$ is assumed to have a finite range $d$, i.e., $V\left(z, z^{\prime}\right)=0$ when either $z$ or $z^{\prime}$ is greater than $d$. Our interest centers on the limit of extremely long chains so that the probability factorizes

$$
\left\langle G\left(\mathbf{r}, n, \mathbf{r}^{\prime}, n^{\prime}\right)\right\rangle \simeq e^{\mu_{0}\left(n-n^{\prime}\right)} \Psi(z) \Psi\left(z^{\prime}\right)
$$

In view of eq 8 , the normalized "wave function" $\Psi(\mathbf{r})=$ $\Psi(z)$ depends on $z$ only. Anticipating that a solution to eq 4 is possible solely in the semidilute region, we let the total number of links $N$ tend to infinity and the magnitude of $\Psi(z)$ go to zero in such a way that $N \psi^{2}(z) \equiv c_{0} \chi^{2}(z)$ remains a finite function. The dimensionless function $\chi$ asymptotes toward unity far from the surface, and $c_{0}$ represents the bulk segment concentration. In addition, we suppose a small interaction exists between the segments denoted by the excluded volume $\beta$. These considerations simplify eq 4

$$
\begin{array}{r}
\mu_{0} \chi(z)-\frac{1}{6} a^{2} \frac{\mathrm{d}^{2} \chi(z)}{\mathrm{d} z^{2}}-c_{0} \xi_{\mathrm{s}}^{2} \chi(z) \int_{0}^{d} V\left(z, z^{\prime}\right) \chi^{2}\left(z^{\prime}\right) \mathrm{d} z^{\prime}+ \\
\beta c_{0} \chi^{3}(z)=0
\end{array}
$$

If the interaction with the surface were absent, we would regain the usual self-consistent field theory ${ }^{5,6}$ with bulk correlation length

$$
\xi_{\mathrm{b}}=\left(\frac{a^{2}}{3 \beta c_{0}}\right)^{1 / 2}
$$

In eq 10 , we naively neglect any coupling between the random averaging and the excluded-volume effect. Preliminary work shows that this is probably reasonable when the excluded volume $\beta$ is small, as will be hypothesized here. This problem will be discussed in a forthcoming paper.

In order to illustrate one type of feasible solution to eq 10 , we simply set $V\left(z, z^{\prime}\right)$ equal to a constant $V$ for both $z$ and $z^{\prime}$ within the range $0-d$. Note that since $\chi \rightarrow$ 1 as $z \rightarrow \infty$, the parameter $\mu_{0}=-\beta c_{0}$ is much smaller than unity when the concentration is semidilute. Thus, the solution to eq 10 in this case is readily obtained by patching the inner to the outer solution at $z=d$. For $z$ 
$>d$, the outer solution is

$$
\chi_{\text {out }}=\operatorname{cotanh}\left(\frac{z+z_{0}}{\xi_{\mathrm{b}}}\right)
$$

whereas for $0 \leqslant z \leqslant d$ the inner solution is approximately given by

$$
\chi_{\text {in }} \simeq A \sin k z
$$

which holds whenever the excluded volume is small enough

$$
\epsilon \equiv \frac{\beta}{d V \xi_{\mathrm{s}}^{2}} \ll 1
$$

The constants $A, z_{0}$, and $k$ can now be eliminated by requiring that $\chi_{\text {in }}^{\prime}=\chi_{\text {out }}^{\prime}$ and $\chi_{\text {in }}=\chi_{\text {out }}$ at $z=d$.

It has been tacitly assumed that the bulk correlation length $\xi_{\mathrm{b}}$ is larger than all other microscopic variables; hence $\xi_{\mathrm{b}} k \gg 1$. Then the algebraic expressions connecting the unknown constants can be solved provided

$$
\epsilon^{1 / 2} \xi_{\mathrm{b}} \gg d
$$

leading to

$$
\begin{gathered}
k d \simeq 1 / 2^{\pi}+\epsilon^{1 / 2} \\
z_{0} \simeq \epsilon^{-1 / 2} d
\end{gathered}
$$

The number of segments adsorbed per unit area is then given $b^{8}$

$$
\sigma=c_{0} \int_{0}^{\infty}\left(\chi^{2}(z)-1\right) \mathrm{d} z \simeq \frac{\epsilon^{1 / 2} a^{2}}{\beta d}=\frac{a^{2}}{d^{3 / 2} \beta^{1 / 2} V^{1 / 2} \xi_{\mathrm{s}}}
$$

As long as inequalities (14) and (15) are both satisfied, it is concluded that the adsorbed amount of polymer increases as the factor $d V \xi_{\mathrm{s}}{ }^{2}$, signifying the effective interaction between a chain segment and the surface decreases. ${ }^{9}$ This peculiarity stems from the strong nonlinearity of the randomly averaged expression for the probability, which can be traced back to the coupling of the stochastic field $\phi$ to $G$ in eq 3 . Our calculation is of course qualitative since both the excluded-volume interaction and the random averaging have been assessed on the selfconsistent field level. Experimentally, the anomalous effect that we predict might be observable for weakly charged polyelectrolytes attracted to an apparently neutral surface at its isoelectric point provided the frozen distribution of remnant positive and negative charges is sufficiently random. Increasing the ionic strength should enhance the polyion adsorption at least within a certain range of added salt.

Just after this work was completed, I became aware of a theory deriving a final expression similar in some respects to those developed here. Garel et al. ${ }^{10}$ use the replica method and other approximations to study the behavior of a random copolymer near the interface between two immiscible liquids. They allow only for a random interaction of the bulk with each segment keeping the Kuhn length fixed. The physics of their system ought to be analogous to that presented here provided we set $\beta$ equal to zero and $V$ equal to a constant of infinite range and also introduce a nonrandom background potential in our analysis. One does indeed regain eq $12 \mathrm{~b}$ of ref 10 instead of our eq 10. A future problem will be to discover why two apparently unrelated formalisms should yield identical results.

Other related works include those on polymer adsorption to sinusoidal gratings ${ }^{11,12}$ and fractal surfaces. ${ }^{13}$ These kinds of geometrical roughness increase the tendency for a polymer to adsorb as does the stochastic roughness introduced here. Hone et al. ${ }^{11}$ also treat stochastic roughness heuristically. Their analysis differs markedly from our treatment since they disregard the stochastic nature of their eigenvalue and average their integral equation in a perturbative fashion analogous to the Keller smoothing method. ${ }^{3}$ Hence, they end up with linear equations that cannot describe the nonperturbative adsorbed state. In our analysis, the eigenfunction expansion is carried out after the random averaging so that the principal eigenvalue is deterministic. Moreover, the random field is coupled nonperturbatively to the propagator, albeit at the self-consistent field level.

\section{References and Notes}

(1) Baumgärtner, A.; Muthukumar, M. J. Chem. Phys. 1987, 87, 3082 .

(2) Edwards, S. F.; Muthukumar, M. J. Chem. Phys. 1988, 89, 2435.

(3) Sobczyk, K. Stochastic Wave Propagation; Elsevier: New York, 1985.

(4) Edwards, S. F. Proc. Phys. Soc. 1965, 85, 613.

(5) Edwards, S. F. Proc. Phys. Soc. 1966, 88, 265.

(6) de Gennes, P.-G. Scaling Concepts in Polymer Physics; Cornell University Press: Ithaca, 1979.

(7) Equation 3 is obtained by a Taylor expansion of the kernel in the recursion relation for the probability $G$ and a linearization of the Boltzmann factor involving the external field (see, e.g.: Lifshitz, I. M.; Grosberg, A. Yu.; Khokhlov, A. R. Rev. Mod. Phys. 1978, 50,683). Actually, the weak field approximation usually implies the Taylor expansion is legitimate. For instance, in the problem at hand, eq 10 shows that the effective attractive potential is at most of order $a^{2} / d^{2}$. Accordingly, we should assume this is a small quantity if the Boltzmann factor is allowed to be linearized, but then eqs 3 and 10 are valid approximations to the original integral equation or recursion relation. In effect, eq 13 proves that $a^{2} \chi^{\prime \prime} \simeq a^{2} \chi /$ $d^{2}$ is much smaller than $\chi$ so that $\chi$ is slowly varying.

(8) As a referee has pointed out, the number of segments adjacent to the surface (proportional to $\sigma(0) \simeq c_{0} d \chi^{2}(z=d) \simeq a^{2}$ / $\left.d^{2} V \xi_{8}{ }^{2}\right)$ is independent of the excluded volume $\beta$. This arises because $\beta$ is a small quantity in the sense of eq 14. Hence, in eq 10 the derivative balances the integral so that the inner layer is relatively unperturbed by the excluded-volume effect and the constant $A \simeq \chi(z=d)$ is independent of $\beta$.

(9) Note that we cannot allow $V$ to become very small because ultimately eq 14 would be violated. Our analysis does not address the conditions under which the chains start to adsorb.

(10) Garel, T.; Huse, D. A.; Leibler, S.; Orland, H. Europhys. Lett. $1989,8,9$.

(11) Hone, D.; Ji, H.; Pincus, P. A. Macromolecules 1987, 20, 2543.

(12) Ji, H.; Hone, D. Macromolecules 1988, 21, 2600.

(13) Blunt, M.; Barford, W.; Ball, R. Macromolecules 1989, 22, 1458. 